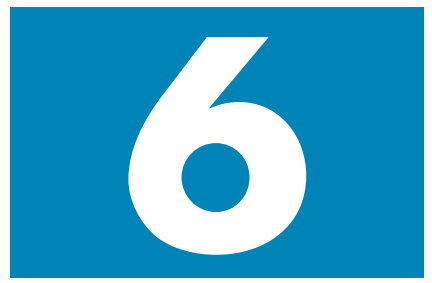

\title{
Orthodontics and occlusion
}

\author{
S. J. Davies, ' R. M. J. Gray, ${ }^{2}$ P. J. Sandler, ${ }^{3}$ and K. D. O'Brien, ${ }^{4}$
}

In this part, we will
discuss:
- What the goals
of orthodontic
treatment are
- The need for a
full articulatory
examination
- Specific treatment
considerations:
Should orthodontic
treatment involve
extractions?
What is meant by
growth modification
and skeletal camou-
flage?
What is the
significance of
tooth drifting?
The relationship
between orthodontic
treatment and
temporomandibular
disorders

\section{What are the goals of orthodontic treatment? \\ - For the \\ Orthodontist \\ - For the Patient \\ Are they the same?}

$1^{*} G D P, 73$ Buxton Rd, High Lane, Stockport SK6 8DR; P/T Lecturer in Dental Practice, University Dental Hospital of Manchester, Higher Cambridge St., Manchester M15 6FH;

${ }^{2}$ Honorary Fellow, University Dental Hospital of Manchester, Higher

Cambridge St., Manchester M15 6FH;

${ }^{3}$ Consultant Orthodontist, Royal Hospital, Calow, Chesterfield S44 5BL;

${ }^{4}$ Professor, University Dental Hospital of Manchester, Higher Cambridge St., Manchester M156FH

${ }^{*}$ Correspondence to : Stephen Davies email:stephen.j.davies@man.ac.uk REFEREED PAPER

() British Dental Journal 2001; 191:539-549

\begin{abstract}
The discipline of orthodontics is directed towards alteration of the occlusion of the teeth and the relationships of the jaws. It is therefore somewhat surprising to find that there is little scientific evidence to support any of the concepts that suggest occlusal goals for orthodontic treatment. Most of the current concepts of orthodontic treatment are based upon personal opinion and retrospective studies. Nevertheless, an attempt is made here to provide a guide to the relationship of orthodontics and the occlusion that is evidence based. Where the evidence is weak, these areas have been highlighted.
\end{abstract}

The goals of orthodontic treatment for the Orthodontist

The orthodontist's goal of treatment is to achieve as near perfect occlusion as possible. But what is perfection and what is this 'optimal occlusion'? If we examine the early orthodontic literature, we will find that the founder of contemporary orthodontics, Edward Angle, attempted to treat his patients' occlusion with reference to the occlusion of a skull displayed on a shelf in his surgery. This skull was affectionately termed 'Old Glory' (Fig. 1a).

There is no doubt that the introduction of, firstly the six keys philosophy (Fig. 1b), and secondly, the pre-adjusted edgewise appliance designed to achieve them was a quantum leap for orthodontists.

Unfortunately, there is no evidence that achieving this 'optimal' occlusion has any influence upon long-term stability, masticatory function or the alleged association between orthodontic treatment and temporomandibular disorders.

The goals of orthodontic treatment for the Patient

The prime reason for a patient to seek orthodontic treatment is to gain an improvement in appearance. Arguably, we should primarily aim to treat the patient's needs rather than pursue a dogmatic adherence to the six keys of occlusion.

\section{Form follows function}

There is fortunately, however, a strong association between the aesthetics of the final treat- ment (patient's goal — Form) and the achievement of an 'optimal' occlusion (orthodontist's goal - Function). This is because if at least keys 2-5 have been achieved, the patient will have perfectly aligned upper and lower front teeth, irrespective of the relationship between the jaws. In Figure 2, the patient has a 'six key occlusion'. In Figure 3, the patient does not have an optimal buccal interdigitation (keys 1 and 2 missing), yet there is very little perceptible difference in the aesthetics of the dentition.

\section{The need for a full examination}

\section{Occlusal examination}

It is important to emphasise that it is necessary to carry out a full occlusal examination for all orthodontic patients. It is essential to record

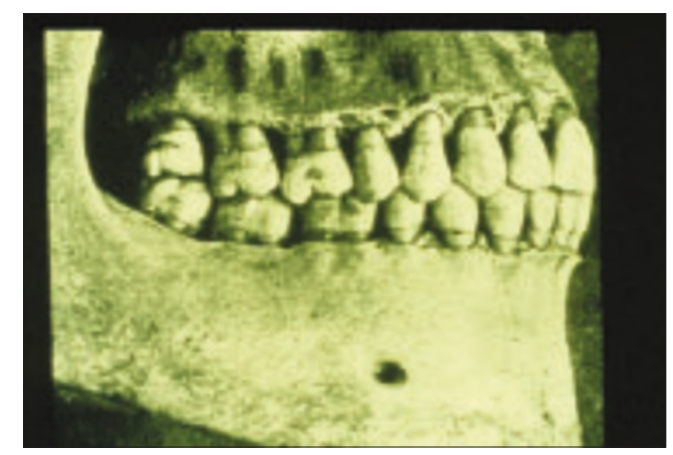

Fig. I a 'Old Glory': Examination of the dentition reveals that this is, indeed, an 'optimal' occlusion. There is a perfect buccal interdigitation, overjet and overbite. These aims were modified by the concepts of Andrews who introduced the 'six keys of occlusion' (see Fig. Ib) 


\section{The concept of treating toward an 'optimal' occlusion is not based on any evidence on the long-term effects of orthodontic treatment}
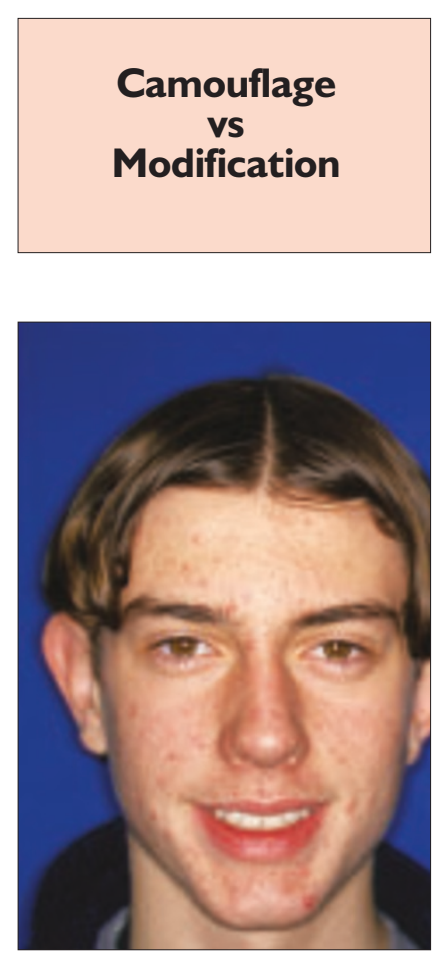

Fig. 2 'Six keys to occlusion' are present (after Andrew ); a perfect smile

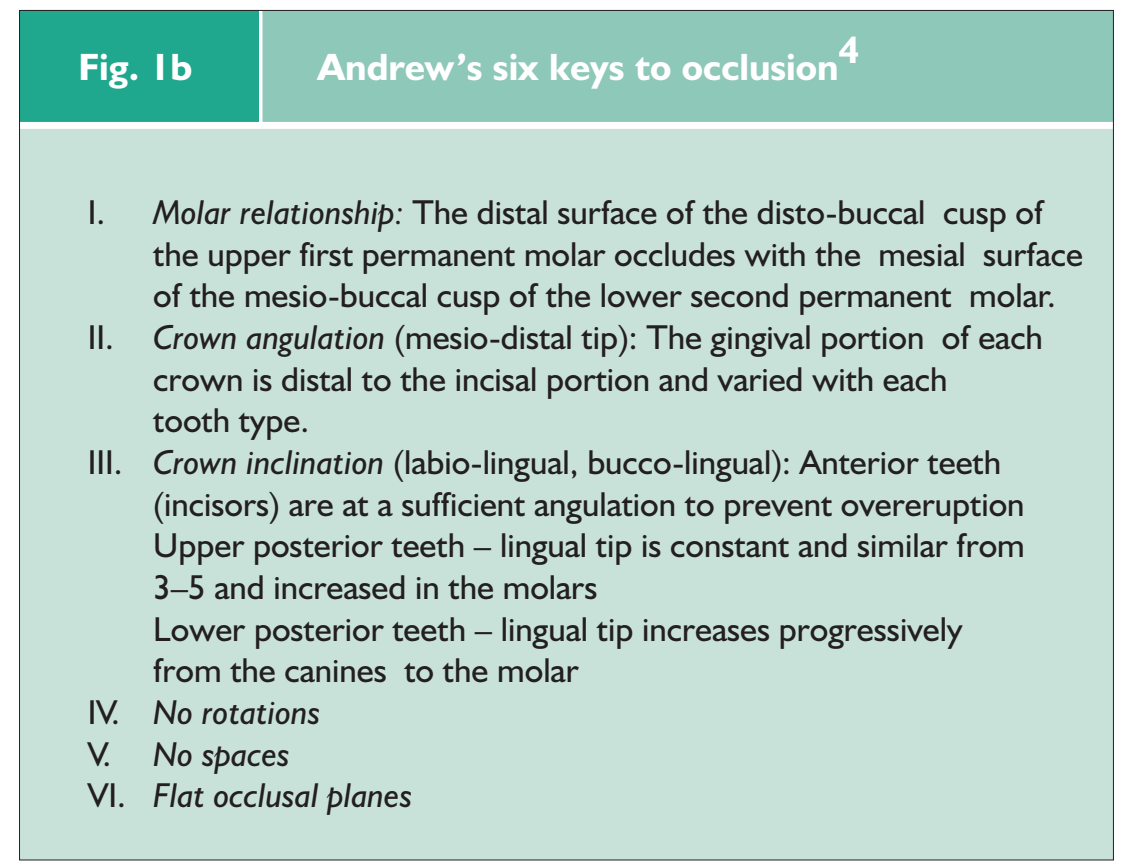

not only the patient's habitual bite (centric occlusion CO or intercuspation position ICP) but also to record the patient's ideal jaw relationship (centric relation $=\mathrm{CR}$ or retruded contact position $=\mathrm{RCP}$ ). This is done against the benchmark of 'ideal occlusion. ${ }^{1}$ Firstly does $\mathrm{CO}$ occur in CR? If not, what is the discrepancy between the two? Secondly does the anterior guidance on the front teeth occur with an absence of posterior interferences?

Without doing this the dentist or orthodontist cannot fully assess a malocclusion or avoid a potential mistake in treatment planning.

This is illustrated in Figure 4. In centric occlusion this patient has an overjet of $5 \mathrm{~mm}$ and appears to represent a relatively straight

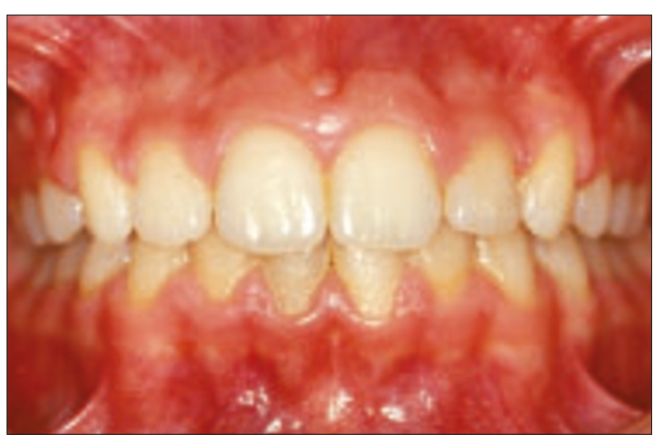

forward orthodontic problem. However, when she is placed into centric relation, the mandible is considerably more posteriorly placed and she has an overjet of $10 \mathrm{~mm}$, which is far more difficult to treat. This is an example of a 'postured bite' and is a relatively common problem in Class II Div i malocclusions. Similar problems may arise from crossbites and premature contacts. As a result, this aspect of an examination is essential if misdiagnosis and incorrect treatment planning is to be minimised.

Examination of the articulatory system

It must be appreciated, however, that the occlusal examination only comprises one part of the articulator system examination; the muscles and temporomandibular joints should also be examined.

The three elements of an examination of the articulator system ${ }^{2}$ can easily be incorporated into an orthodontic examination protocol (Fig. 5a-c). This comprehensive examination is divided into two main parts. The jaw and facial examination will record the pattern of the skeletal bases and the facial symmetry, the condition of the TMJs and mandibular muscles, and the soft tissue characteristics. The dental and occlusal examination will record the position of the teeth and their occlusal contacts.

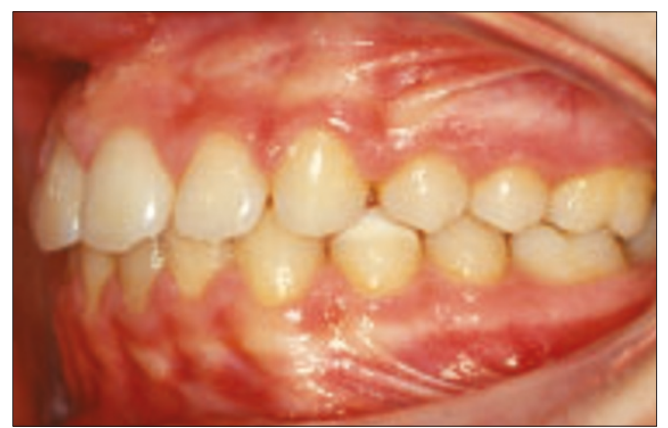

\section{Treatment considerations}

Orthodontic treatment methods can be divided into two depending on the goals of treatment, ${ }^{3}$ these are camouflage and modification. To introduce this concept we must first consider the aetiology of a malocclusion, which includes skeletal, dento-alveolar and soft tissue components, the first two compo- 
nents being particularly relevant. An example of the dento-alveolar component is crowding as a result of an adverse tooth-alveolar tissue ratio. When the malocclusion is caused by the skeletal component, the patient typically presents with a discrepancy between the size or position of his or her skeletal bases in the anterio-posterior, lateral or vertical dimension.

At a very simplistic level the orthodontist when faced with these discrepancies has two main choices:

- To provide treatment directed at camouflaging the problem and not changing the skeletal pattern.

or

- Attempting skeletal modification with either functional appliances or orthognathic surgery

\section{Camouflage}

If the treatment is directed at camouflage, space is created in the dental arches by extractions, arch expansion or both, and the other teeth are moved into this space to achieve the treatment objective.

Even if the person has a skeletal discrepancy, the orthodontist may choose to accept the discrepancy and attempt to camouflage the problem by simply moving the teeth.

Unfortunately, whilst the dental appearance can be predicted the facial appearance cannot (Figs 6 and 7). Furthermore, there is no scientific evidence for any technique that may aid in prediction. Some clinicians may say they can accurately predict facial changes, but this claim is totally unfounded.

\section{Modification}

An alternative treatment is to attempt to change the skeletal pattern. This may be achieved by 'growth modification' which comprises the use of functional appliances in the growing child pattern (Fig. 8) or by orthognathic surgery, in the adult pattern (Fig. 9).

How does the orthodontist decide on attempting camouflage or growth modification therapy?

There is currently a debate in orthodontics concerned with the detrimental effect of camouflage on the facial profile. Many orthodontists attempt growth modification treatment for children instead. Surprisingly, there is no evidence that functional appliances alter the skeletal pattern of the growing child in any significant way. ${ }^{5}$ A recent investigation has shown that most of the change arising from Twin Block treatment is dental tipping with only a small contribution from a change in the jaw relationship. ${ }^{6}$ It is still not known if such treatment changes the size and shape of the jaws or purely the relationship of the upper to the
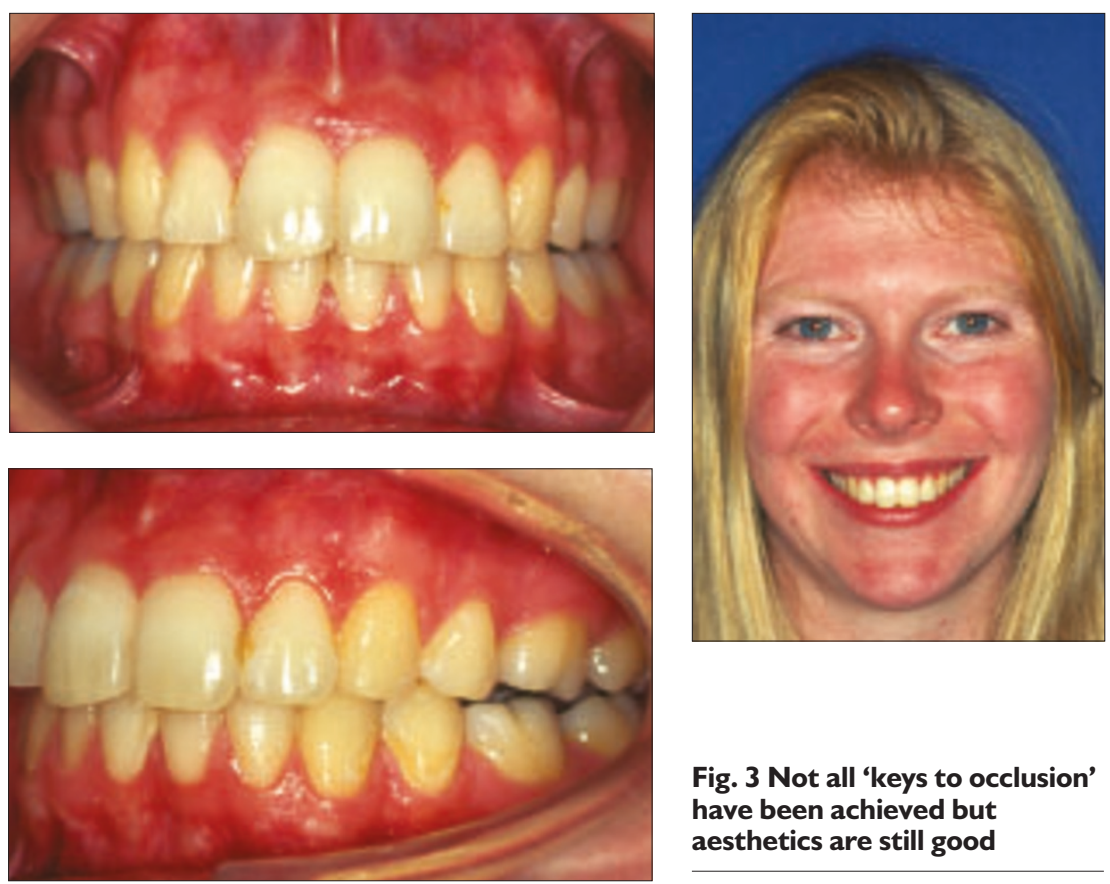

Fig. 3 Not all 'keys to occlusion’ have been achieved but aesthetics are still good

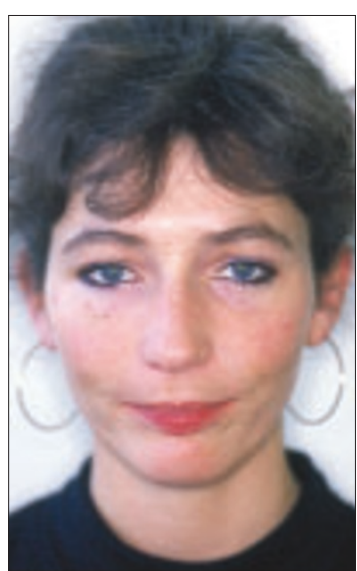

\section{Centric Relation}

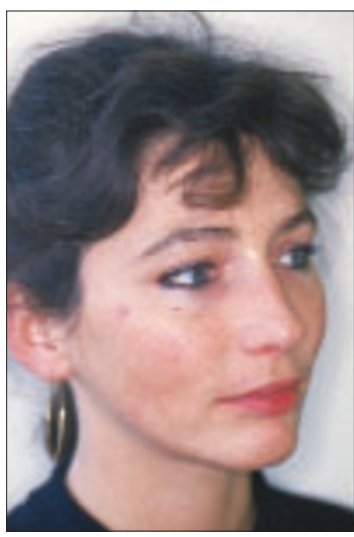

Centric Occlusion

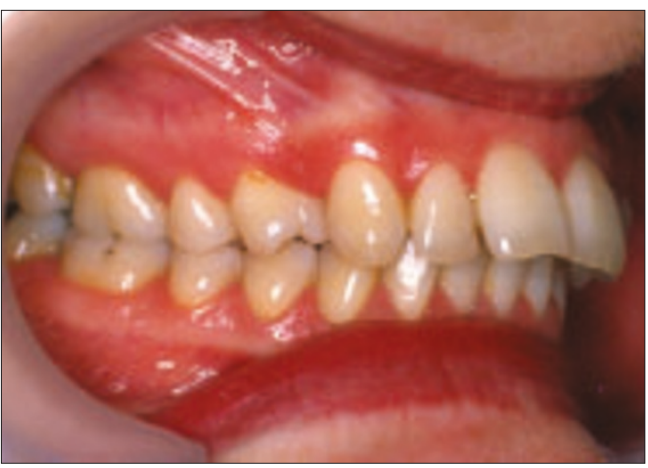

Centric Relation

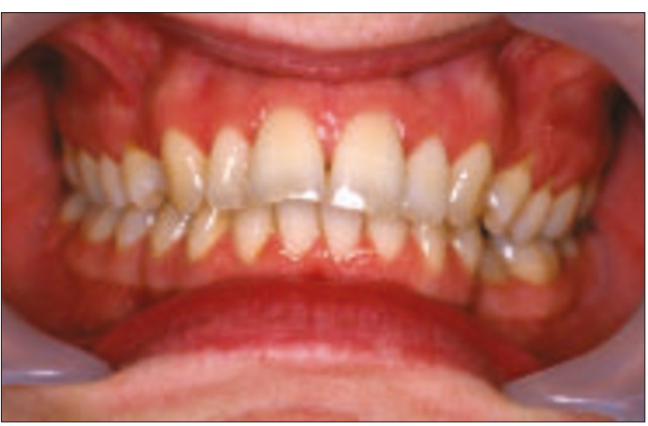

Centric Occlusion

Fig. 4 A patient who has a $5 \mathrm{~mm}$ overjet when occluding in centric occlusion and has a $10 \mathrm{~mm}$ overjet when in centric relation 


\section{Fig. 5a}

Orthodontic examination including articulatory system exam .... Page I

\section{General Record}

Patient

Date

Age

Medical

history

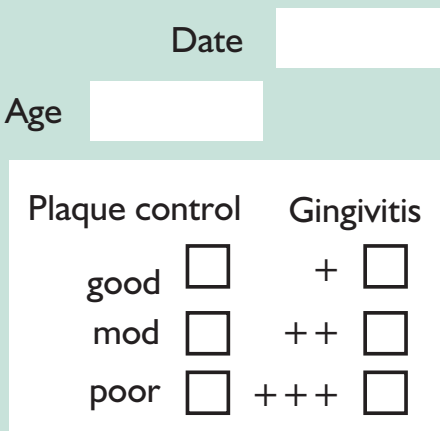

Jaw and Facial Examination

Skeletal Pattern I II III $\quad$ mild mod severe
TMJ
Tender to palpation?

Noises

Clicks

Right Left or Bilateral

Soft or Loud

Consistent or Intermittent

Opening or closing or both

Early Mid Late

Painful or Painless

Single or Multiple

Crepitus Right Left or Bilateral

Painful or Painless

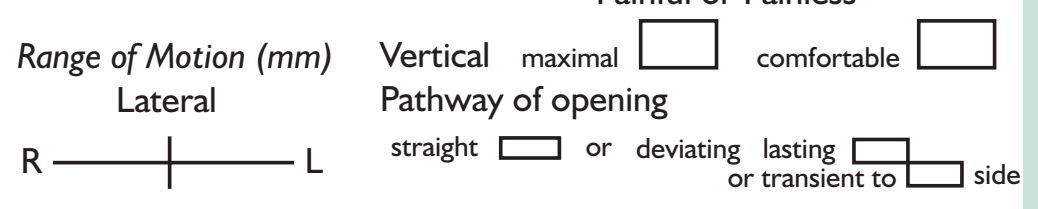

\section{Muscle Tenderness}

Temporalis Masseter

Lateral Pterygoid

Lips Comp $\square \quad \begin{aligned} & \text { Tongue } \\ & \text { thrust }\end{aligned} \square$ Incomp

\section{Centre Lines}

midface

$U$

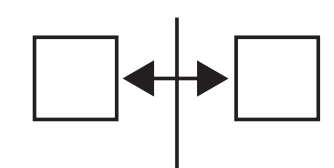

L

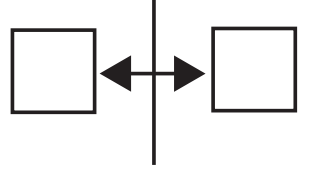


Dental and occlusal examination

Erupted teeth

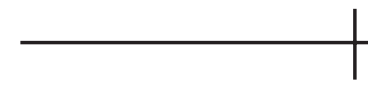

Unerupted teeth

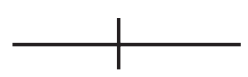

Upper arch

Crowding Inclination
mild $\square$ none $\square$ proclined $\square$
mod $\square$ spaced $\square \quad$ AV $\square$
sev $\square$ fraenum $\square$ retroclined $\square$

Absent teeth

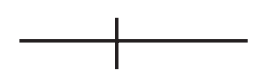

Lower arch

Crowding Inclination

mild $\square$ none $\square$ proclined

$\bmod \square$ spaced $\square \quad$ AV $\square$

$\operatorname{sev} \square$ fraenum $\square$ retroclined $\square$

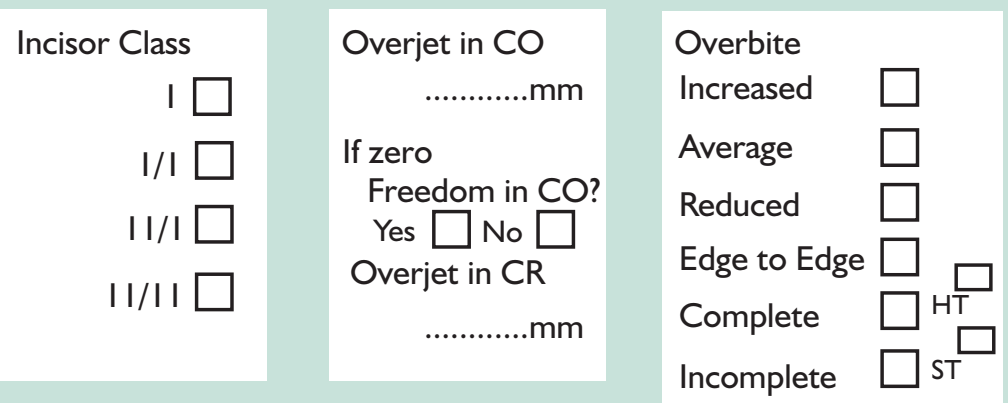

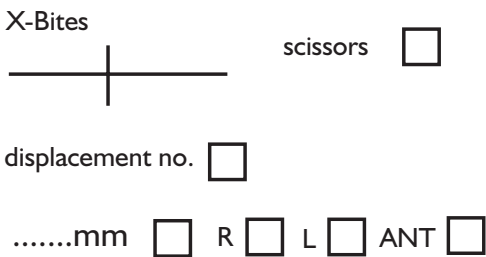

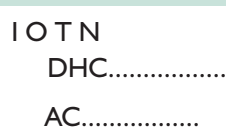

Page 2
If there is a possibility of changing the jaw relationship, the clinician should examine the patient's jaw relationship before and after treatment 
Dental and Occlusal Examination cont.

Buccal Segments

Molars

$\mathrm{I}$
$\mathrm{R} \square$....unit
$\mathrm{L} \square$.....unit $\square$

Canines

$\mathrm{R} \square$....unit $\quad$ II III

$\mathrm{L} \square \quad$.....unit $\square \square$

\section{Static Occlusion}

Does $\mathrm{CO}$ occur in $\mathrm{CR}$ ?

IF NOT..prem contact in CR?

Roughly or exactly

Direction of slide from $C R$ to $C O$

\section{Dynamic Occlusion}

Non-Working Side Interferences

RHS LHS

Working Side Interferences

Crossover Position NWS Int

WS Int

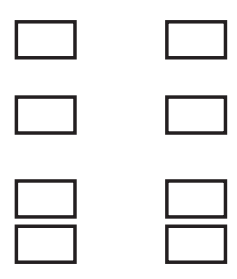

Canine Guidance

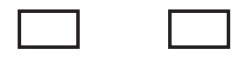

Group Function 
lower jaw. This type of treatment, if it works, does provide excellent dental results (Fig. 8) and, perhaps, reduces the overall time that a child spends wearing fixed appliances.

Orthognathic surgery obviously alters a person's skeletal pattern, however, this is a very complex specialist treatment that should only be carried out, after counselling, for those patients who have major concerns with their facial and dental appearance.

One other form of 'growth modification' is expansion of the dental arches with either removable or fixed appliances. This type of treatment is gaining popularity with many dentists and orthodontists, especially those who attend courses of the latest 'orthodontic guru'.

There is, however, no evidence that these appliances can alter the growth of the alveolus and some research has suggested that most expansion is not stable and simply invites relapse in the occlusion. ${ }^{7}$

\section{Extraction vs non-extraction}

There has been considerable research into the potential 'harmful' effect of extractions on the dentition, the face and occlusion but it must be emphasised that no scientific study has concluded that orthodontic extractions are detrimental. Unfortunately, the myth still persists and the orthodontists who suggest this type of conventional treatment are frequently described as 'narrow minded' and those who 'do not know any better'.

If a thorough appraisal of the scientific literature is undertaken no evidence of the deleterious effect of extractions can be found.

\section{Orthodontics and temporomandibular disorders (TMD)}

This link, as always, courts considerable controversy. As discussed there is a tendency to be critical of tooth extraction as part of orthodontic treatment. Implicit in this is the feeling that orthodontic extractions adversely affect the occlusion and furthermore this then leads to a temporomandibular disorder. First premolar extractions are considered by some to be an aetiological factor in TMD, it is suggested that these extractions permit the posterior teeth to move forward resulting in a decrease in the vertical dimension of occlusion. ${ }^{8}$

Another theory is that first premolar extractions lead to over-retraction of the anterior teeth, particularly the maxillary incisors. The anterior teeth then displace the mandible and the condyles posteriorly. Again, it is hypothesised that this may lead to a TMD. ${ }^{9}$ These theories may superficially appear plausible. When, however, they are subjected to scientific scrutiny they do not satisfy even the most basic criteria of scientific investigation. The 'evidence' that is commonly quoted is derived purely from personal opinion. Until randomised clinical tri-
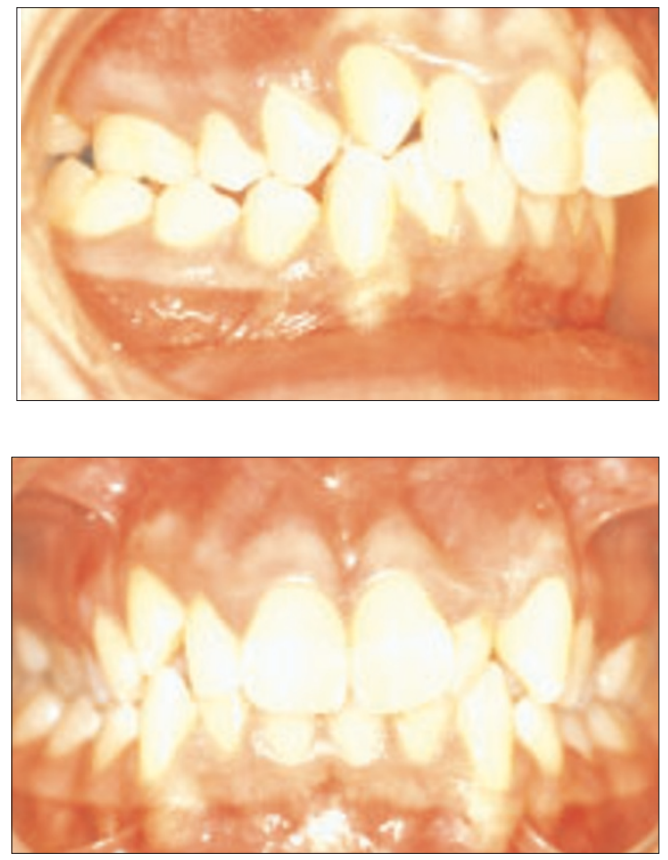

Fig. 6a shows the patient before treament
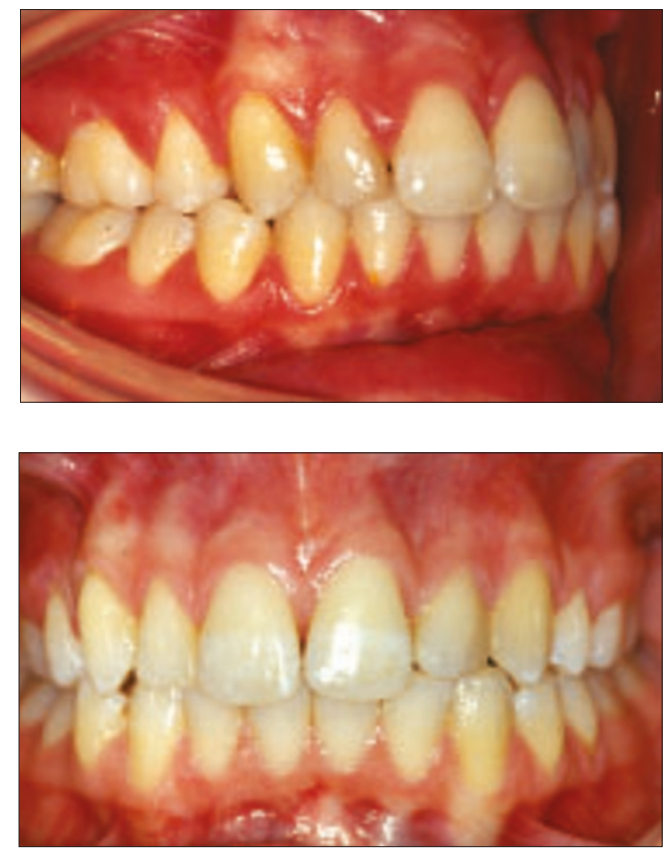

Fig. 6b shows the patient after 'camouflage' treatment. There is a good facial and dental appearance
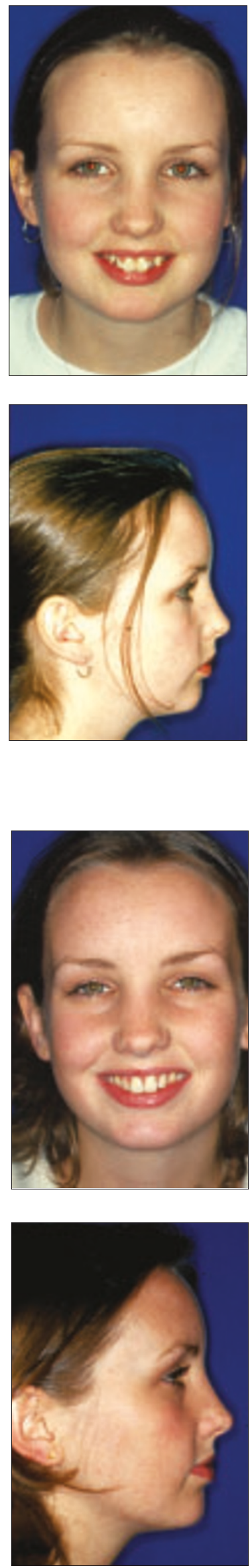


\section{PRACTICE occlusion}
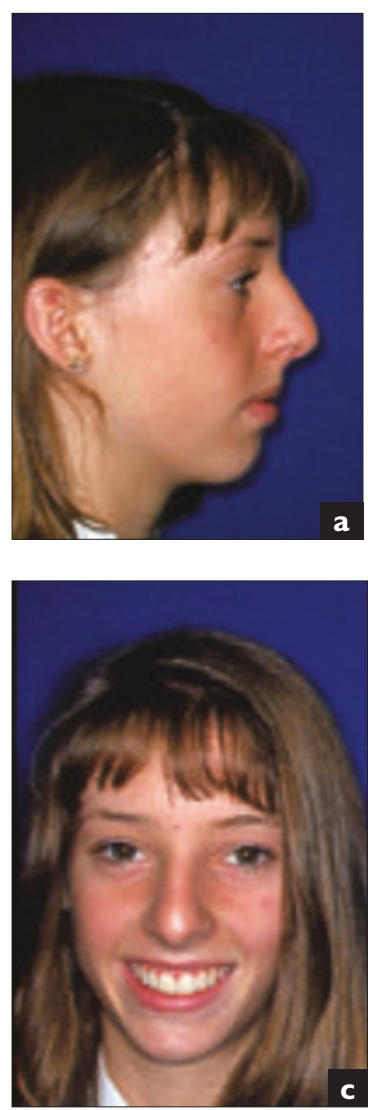

Fig. 7 A Cl 2 div ii following 'camouflage' treatment; showing good occlusion (7b, 7d), but disappointing facial appearance (7a, 7c)
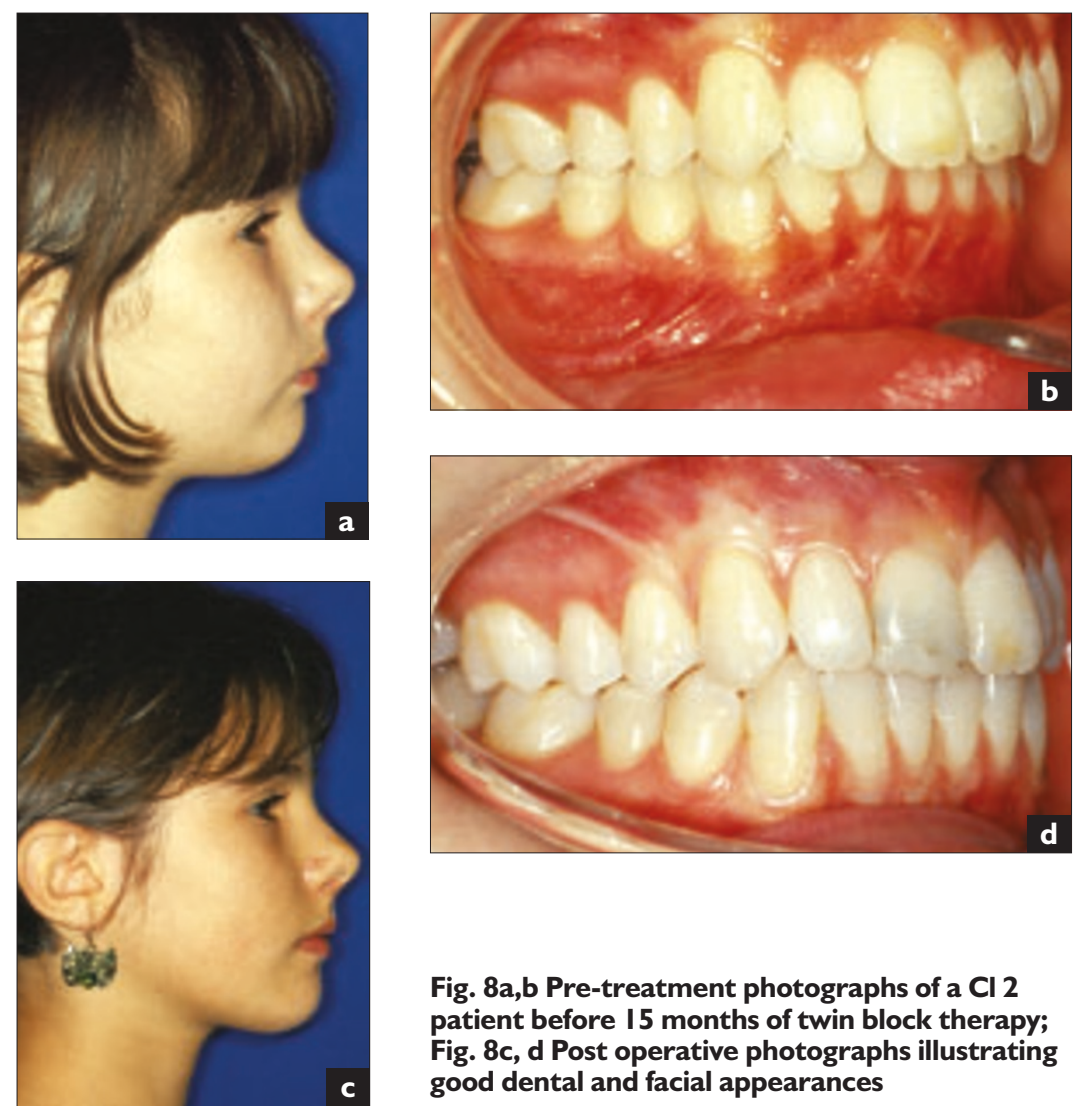

als have been carried out these theories must remain as simply conjecture, anecdotal and lacking in evidence.

Orthodontics and temporomandibular disorders?

Does orthodontic treatment cause TMD? Clinical experience and review of the literature does not support any aetiological relationship.

Can orthodontic treatment cure TMD?

Occasionally orthodontics will form some part of the treatment plan.

\section{Summary}

The relationship between orthodontics and occlusion is important. Unfortunately, supposition and hearsay can govern treatment philosophy.

Confining considerations to factors that are supported by scientific evidence, the following conclusions may be accepted:

1. The aims of orthodontic treatment are, surprisingly, unclear. On the one hand an argument can be made for providing a 'perfect or optimal occlusion'. There is little evidence in the literature to suggest that this is necessary. Some features of ideal occlusion do, however, provide optimal aesthetics. This introduces the alternative treatment aim to provide the patient with an appearance that is acceptable to society's aesthetic norms.

2. Acceptable orthodontic treatment goals (including a good occlusion) can be achieved by either the camouflage or skeletal modification approach.

3. Extraction of teeth is needed as a part of many courses of orthodontic treatment. When carried out as a part of a comprehensively planned course of orthodontic treatment, no damage is done to the facial profile or occlusion.

4. There is no evidence in the literature that a treatment plan involving the extraction of teeth is more prone to relapse or predisposes the development of a TMD.

5. An examination of the patient's static occlusion must not only include assessment of the habitual bite or centric occlusion (CO), but also the occlusion in centric relation (CR). This is because the presence of large discrepancies between CO and CR are a positive indication for orthodontic treatment. Equally, such discrepancies should not be introduced during orthodontic treatment. In restorative terminology orthodontic treatment is a 're-organised' treatment plan; and to leave or create a large discrepancy between $\mathrm{CO}$ and CR would be to provide the patient with a less than ideal occlusion. 

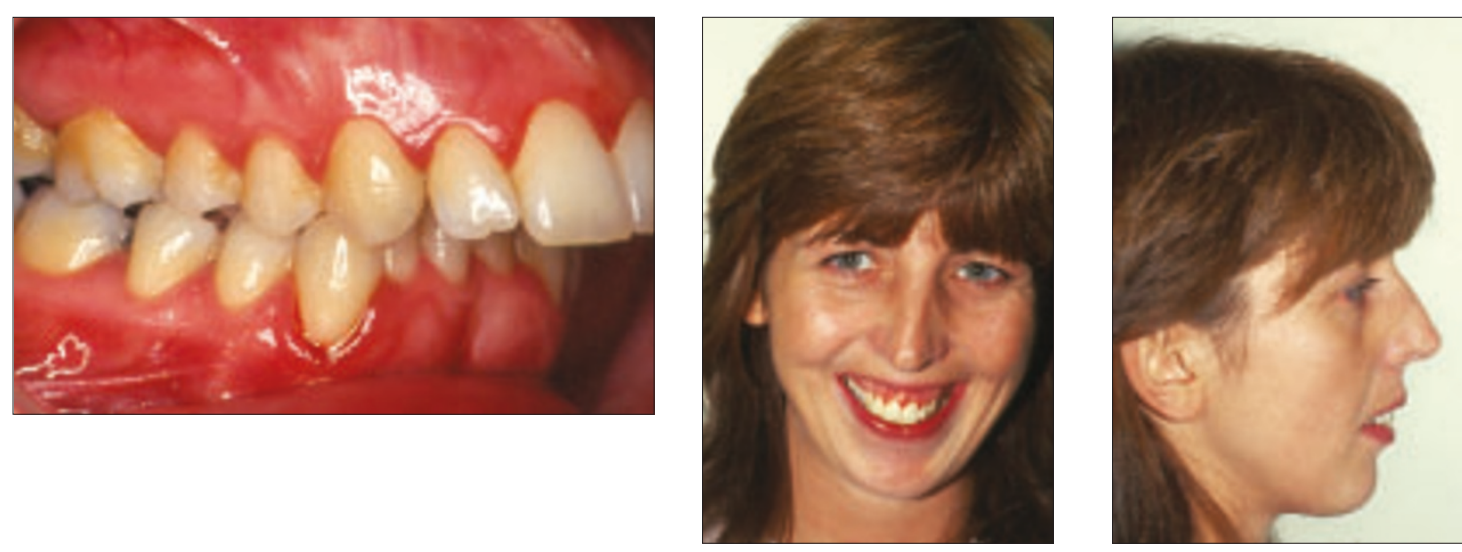

Fig. 9a i -iii Pretreatment photographs of a $\mathrm{Cl} 2$ patient before orthognathic surgery
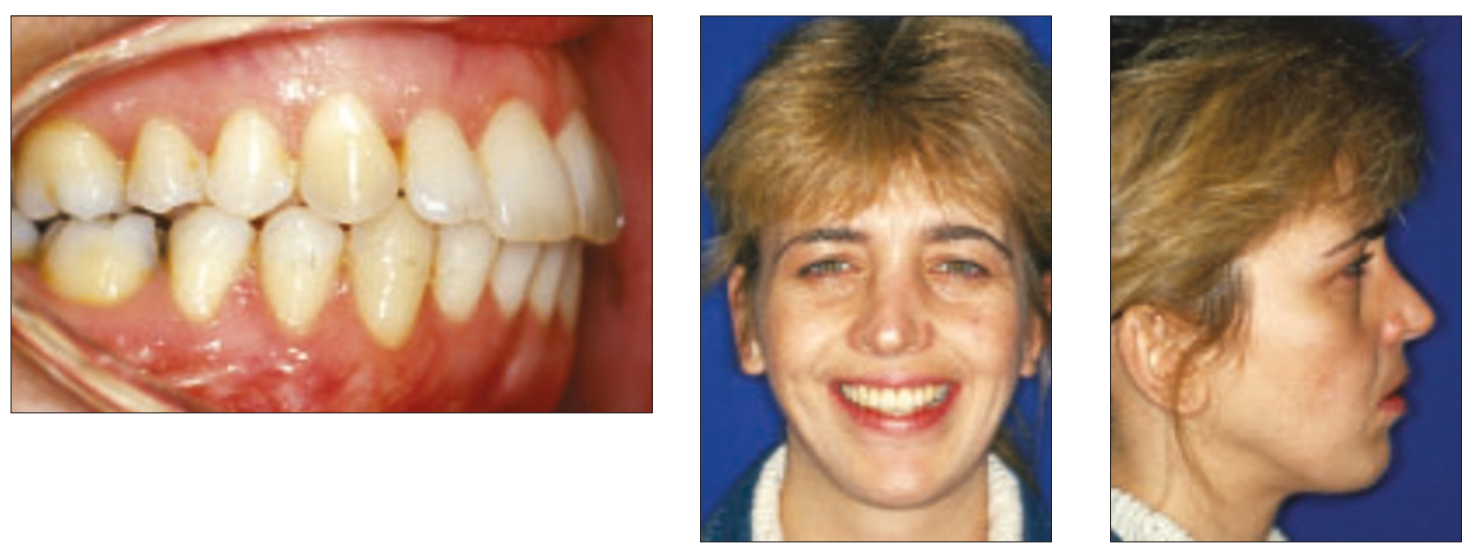

Fig. $9 \mathrm{~b}$ i-iii Post operative photographs illustrating good dental and facial appearances

\section{Guidelines of good occlusal practice}

I The examination of the patient involves the teeth, periodontal tissues and articulatory system.

2 There is no such thing as an intrinsically bad occlusal contact, only an intolerable number of times to parafunction on it.

3 The patient's occlusion should be recorded, before any treatment is started.

4 Compare the patient's occlusion against the benchmark of ideal occlusion.

5 A simple, two dimensional means of recording the patient's occlusion before, during and after treatment is an aid to good occlusal practice.

6 The conformative approach is the safest way of ensuring that the occlusion of a restoration does not have potentially harmful consequences.

7 Ensuring that the occlusion conforms (to the patient's pre-treatment state) is a product of examination, design, execution and checking (EDEC)

8 The 'reorganised approach' involves firstly the establishment of a 'more ideal' occlusion in the patient's pretreatment teeth or provisional restorations; and then adhering to that design using the techniques of the 'conformative approach'

9. An 'ideal occlusion' in removable prosthodontics is one which reduced de-stabilisating forces

10. The occlusal objective of orthodontic treatment is not clear, but a large discrepancy between centric occlusion and centric relation should not be an outcome of treatment

II. An 'orthodontic' examination of the occlusion should include: the dynamic occlusion; and the jaw relationship in which the patient has centric occlusion
1 Ash M M, Ramfjord S P. Occlusion 4th ed. pp84-85, Philadelphia: Saunders, 1995

2 Gray R M J, Davies S J, Quayle A A. Temporomandibular disorders: a clinical approach, pp9-25.BDJ publications, 1995, 1997.

3 Proffit W R. Contemporary orthodontics, 1st ed. London: Mosby, 1986, p212.

4 Andrew L F. The straight wire appliance. BrJ Orthod 1979; 6: 125-143.

5 Tulloch J F C, Philips C, Koch G, Proffit W R. The effect of early intervervention on skeletal pattern in Class II malocclusion; a randomised contrlled trial. Am J Orthod 1997; 111: 391-400.

6 Lund D I and Sandler P J.The effect of twin blocks. A prospective controlled study. Am J Orthod 1998; 113: 104-110.

7 DelaCruz A, Sampson P, Little R M, Artun J, Shapiro P A. Long term changes in arch form after orthodontic treatment and retention. Am J Orthod 1995; 107: 518-530.

8 Witzig J W, Spahl T J. The clinical management of basic maxillofacial orthodpaedic appliances. Littleton, Mass.: PSG Publishing Co Inc, 1987.

9 Fennell M, Frost S. Dentofacial orthopaedics and the GDP Part 3. Dent Prac 1993; 31: 8-9. 